\title{
Field Testing of Utility Robots for Lunar Surface Operations
}

\author{
Terrence Fong, Maria Bualat, Matt Deans*, \\ Mark Allan, Xavier Bouyssounouse*, Michael Broxton, Laurence Edwards, \\ Rick Elphic, Lorenzo Flückiger, Jeremy Frank, Leslie Keely, Linda Kobayashi*, \\ Pascal Lee, Susan Y. Lee, David Lees*, Estrellina Pacisł Eric Park* \\ Liam Pedersen*, Debra Schreckenghost $\ddagger$ Trey Smith, Vinh To, and Hans Utz* \\ *NASA Ames Research Center, Moffett Field, CA 94035, USA \\ ${ }^{\dagger}$ Space and Naval Warfare Systems Center, San Diego, CA 92152, USA \\ ${ }^{\ddagger}$ TRACLabs, Inc., Houston, TX r7058, USA
}

\begin{abstract}
Since 2004, NASA has been working to return to the Moon. In contrast to the Apollo missions, two key objectives of the current exploration program is to establish significant infrastructure and an outpost. Achieving these objectives will enable long-duration stays and long-distance exploration of the Moon. To do this, robotic systems will be needed to perform tasks which cannot, or should not, be performed by crew alone. In this paper, we summarize our work to develop "utility robots" for lunar surface operations, present results and lessons learned from field testing, and discuss directions for future research.
\end{abstract}

\section{Introduction}

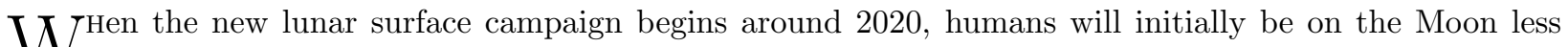
W than $10 \%$ of the time. During the $90 \%$ of time between crew visits, however, robots will be available to perform surface operations. A central challenge, therefore, is to develop robotic systems that can work effectively between (and during) human missions, in order to reduce risk and improve productivity.

For example, site surveys will need to be carried out at a variety of locations, including on rugged terrain and in permanently shadowed regions. Site surveys will involve instruments to characterize the surface and subsurface, to prospect for resources, and to plan science operations. Performing these surveys, particularly with dense coverage, will require hundreds of measurements and hundreds of hours of survey time. Such a tedious task would be highly unproductive for crews to perform alone.

To address this need, we are developing teleoperated and supervised "utility robots" to perform routine, repetitive, and long duration tasks that would be unproductive for crew to manually perform. Our approach is to automate low-risk, surface operations that do not require robots to operate in close, physical proximity to EVA crew and that do not require robots to work at a human pace, or under continuous control.

Utility robots can significantly enhance planetary surface exploration, working in a complementary manner to humans. This is especially true when crew time is limited, or when crews are required to operate independent of ground control. Utility robots can operate prior to crew arrival, performing advance work (e.g., reconnaissance) to improve human productivity. Utility robots can also operate after crew departure, performing follow-up work (e.g., sample curation) or to complete tasks.

During the past three years, we have developed utility robots for four tasks that we believe are critical for sustained lunar exploration. These tasks are: visual inspection - acquiring imagery of sufficient resolution and dynamic range to assess equipment (e.g., crew rover) integrity; systematic site survey - dense area mapping for site characterization and engineering; resource prospecting - detecting and localizing nearsurface hydrogenous deposits for in-situ resource utilization; and robotic reconnaissance - scouting, triage and prioritization of sites/targets of interest to plan crew traverses.

*terrence.w.fong@nasa.gov 


\section{K10 Planetary Rovers}

In our work, we use two third-generation "K10" planetary rovers (Figure 1). Each K10 has fourwheel drive and all-wheel steering with a passive rocker suspension. This design allows operation on moderately rough natural terrain at human walking speeds (up to $90 \mathrm{~cm} / \mathrm{s}$ ).

K10 has hard mounting points on the front, back, and bottom of the body as well as a $100 \mathrm{~cm}$ high mast. This allows attachment of additional components including antennas, navigational sensors, science instruments and mechanical equipment.

K10's avionics design emphasizes off-the-shelf components and modules. The robot runs off of twenty-four $14.4 \mathrm{~V}, 6.6 \mathrm{AH}$ Li-Ion smart battery packs. K10's controller runs on a Linux-based laptop and communicates via $802.11 \mathrm{~g}$ wireless. ${ }^{1} \mathrm{~K} 10$ 's

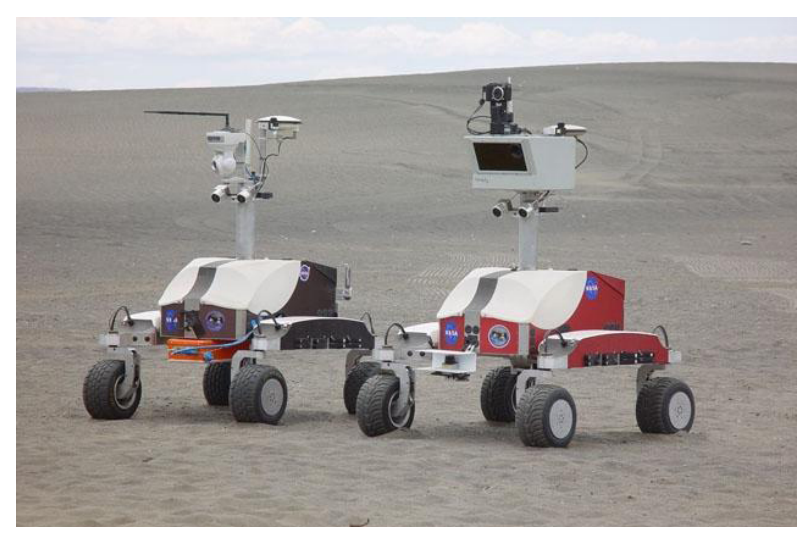

Figure 1. K10 "Black" and "Red" planetary rovers equipped with a variety of science instruments. standard sensor suite includes a Novatel differential GPS system, a Honeywell digital compass, Firewire stereo cameras, a Hetronics or Omnex wireless e-stop, a suntracker, and wheel encoders.

The K10 controller is based on a Service-Oriented Architecture (SOA). ${ }^{2}$ Each component in this architecture provides specific functionality and exposes a clear interface to the others. The interfaces are defined using the Interface Definition Language (IDL) ${ }^{3}$ and communication between components relies on CORBA. ${ }^{4}$ Examples of major components include: locomotion, localization, navigation, and panorama acquisition. Many of these components are built using facilities provided by the CLARAty (Coupled Layer Architecture for Robot Autonomy) framework. ${ }^{5}$

Each subsystem is a service component which only relies on abstract (IDL-defined) interfaces to other components, which are resolved at service startup time. The SOA allows us to group the services into dynamic libraries that can be loaded and configured at run time. This reduces development time as well as memory requirements. The highly decoupled nature of the system also facilitates removal of deprecated services with minimal effort.

\section{Visual Inspection}

In 2006, we developed a system to perform a remote "walk-around" visual inspection of the NASA Johnson "SCOUT" astronaut rover (Figure 2). ${ }^{6}$ Our goal was to provide a human operator (remotely located in a habitat or at ground control) with imagery of sufficient resolution and dynamic range to assess vehicle integrity and detect surface damage.

Using robots to perform remote inspections is one way of reducing risk to humans during exploration missions by reducing the number and duration of EVA's. However, in order for remote inspection to be effective, the system must be sufficiently easy to use, so that mission operations are simple and efficient.

In our approach, K10 automatically collected high-resolution, high-dynamic range (HDR) im-

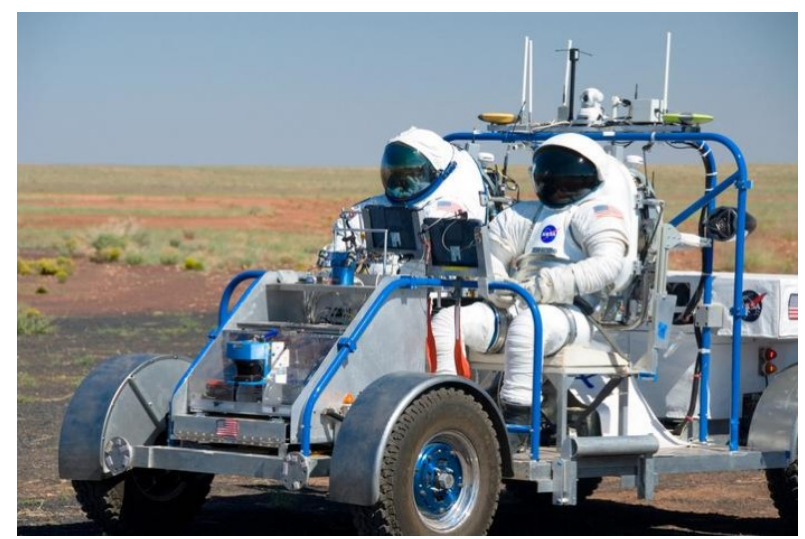

Figure 2. SCOUT is a prototype astronaut rover.

agery from multiple locations around SCOUT. This imagery was then transmitted to the operator station, where they were stitched into a multi-resolution panoramic image. A custom Panorama Viewer enabled operators to interactively explore the panoramas. 


\section{A. Inspection Task Execution}

To collect inspection data, we defined primary and secondary inspection stations relative to SCOUT. K10 was then instructed to drive to each of these station, acquire a panoramic image of SCOUT from that viewpoint and send it to operator station. Primary inspection stations were specified as mandatory, while secondary stations were to be performed only if the rover was operating well within a specified time limit.

We encoded the inspection task using the PLan EXecution Interchange Language (PLEXIL). ${ }^{7}$ PLEXIL is a language for representing control plans that is expressive and deterministic, and which has formal semantics enable verification and validation to. ${ }^{8}$ Task execution was performed using the lightweight "Universal Executive", which is designed to efficiently interpret PLEXIL plans and handle contingencies. ${ }^{7}$

\section{B. HDR Imaging}

The range of brightness values in most outdoor scenes far exceeds the dynamic range of commodity CCD and CMOS sensors. The luminance of a sunlit scene may vary by five (or more) orders of magnitude, whereas most solid-state sensors are 12-bit devices with a limited dynamic range of three orders of magnitude. Hence, even when the optimal exposure is measured and set for the scene, most images captured in a sunlit environment contain some regions that are either over- or under-exposed.

We addressed this issue by capturing a bracket of several images with varying exposures. A bracket consists of images with evenly spaced exposures that range from underexposed (to capture bright regions) to overexposed (to capture dark regions). The number of images in the bracket can be increased until the full dynamic range of the scene has been captured.

Once captured, the bracketed images are combined into a single HDR image using the weighted averaging technique of Reinhard. ${ }^{9}$ Combined in this manner, a HDR image contains well-exposed pixels for every part of the image.

\section{Panorama Display}

We developed a custom user interface, the "Panorama Viewer", to allow interactive exploration of highresolution panoramas. The "Panorama Viewer" was designed to facilitate smooth navigation (zooming and panning) of large panoramas with multiple levels of detail. In addition, it enabled users to quickly switch between panoramas acquired at adjacent inspection stations. Both of these features helped provide continuous contextual cues to operators.

We implemented the "Panorama Viewer" using the NASA Ames "Viz" 3D visualization system, ${ }^{10}$ which was designed for simulation and operation of planetary rovers. The current version of Viz is written in Java and runs as a "plug-in" component in the NASA Ensemble mission operations software framework. ${ }^{11}$

\section{2006 Field Test at Meteor Crater}

We tested our visual inspection system in September 2006 as part of a field test at Meteor Crater, Arizona. ${ }^{12}$ This field test involved researchers from several NASA Centers and examined surface operations that might occur after a crew returns from an EVA sortie to a lunar outpost. The NASA Johnson SCOUT was used as a proxy for an unpressurized crew rover.

During the field test, pressure suited test subjects parked SCOUT within a designated inspection area at the conclusion of each simulated sortie. Once the suit subjects egressed from the vehicle, we commanded K10 to begin its inspection. In total, we performed 18 inspection runs and collected both low-dynamic range (LDR) and HDR panoramas over approximately 25 hours of operation.

We captured high-resolution (12,000 x 6,000 pixels) inspection panoramas using a 2 Mpix $(1,600 \times 1,200$ pixel) digital camera mounted on a motorized pan-tilt head. For each panorama, we acquired images from 54 pan-tilt positions, which covered a $90^{\circ}$ (horizontal) $\times 40^{\circ}$ (vertical) field of view. LDR panoramas required only a single, fixed exposure at each pan-tilt position, for a total of $100 \mathrm{Mpix} /$ panorama. HDR panoramas required a bracket of five exposure levels at each pan-tilt position, for a total of $500 \mathrm{Mpix} /$ panorama.

Figure 3 shows a comparison of LDR and HDR inspection panoramas. The HDR panoramas contained sufficient resolution to enable a very detailed inspection of the SCOUT. Small details that would normally be obscured by deep shadow, such as a pebble stuck in the tread of the tire under the wheel well, were clearly visible. For future operations on the lunar surface, where stark illumination routinely poses a problem, this type of imaging will be extremely valuable. 


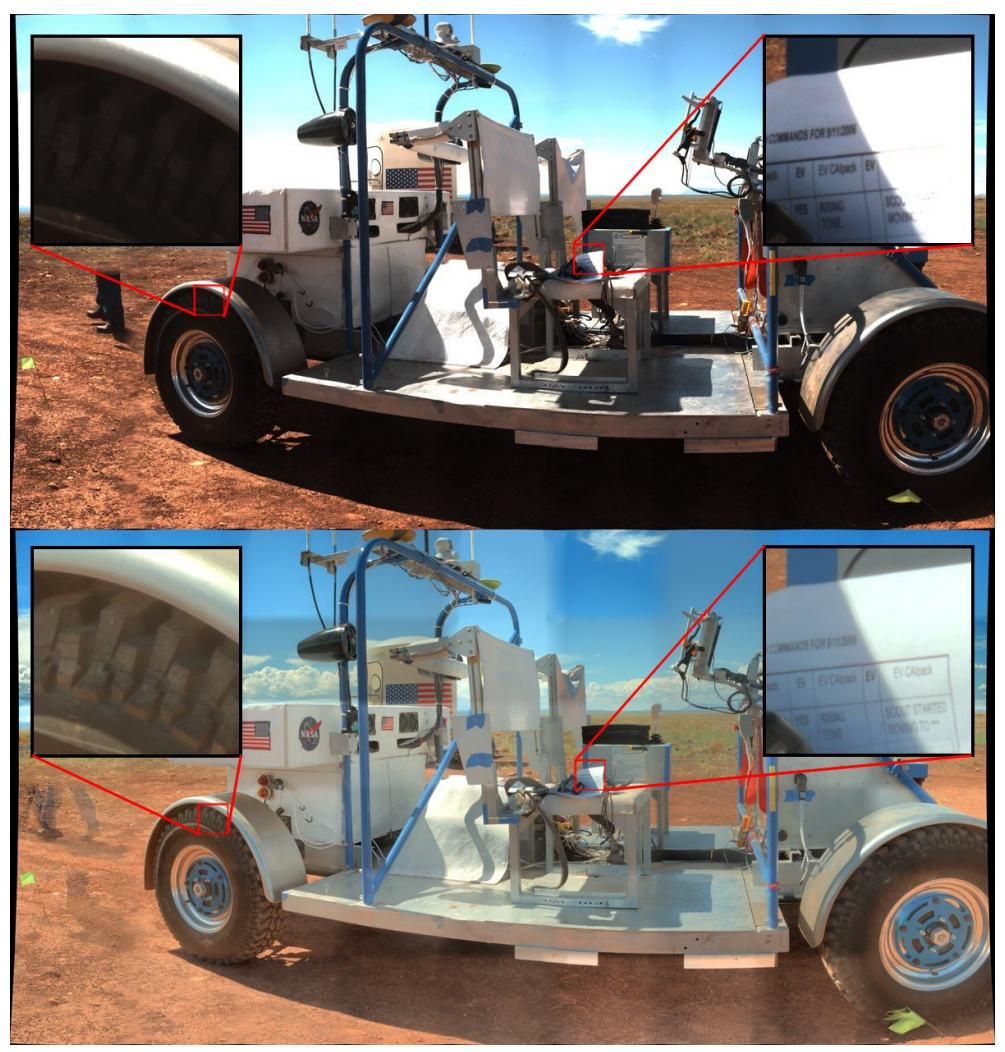

Figure 3. Inspection panoramas: top, LDR image; bottom, HDR image. The HDR image shows numerous features in deep shadow and bright highlight that are not visible in the LDR image.

\section{Systematic Site Survey}

Sustained lunar exploration will require detailed mapping. These surveys will be used for site planning (landing zones, infrastructure installations, etc.), for resource prospecting (minerals and volatiles), and for a variety of lunar surface science investigations. Although data acquired from lunar orbiters will provide wide area coverage, resolution will typically be on the meter to kilometer scale. Smaller-scale variations, groundtruthing, and contact measurements, however, can only be documented by systematic surface surveys.

The difficulty with systematic surveys is that hundreds (or thousands) of repetitive measurements may need to be made at precise locations or along specific trajectories. Collecting this data will be tedious, will require significant driven distance (e.g., parallel-line transects), and will take time. As a result, it would be unproductive for crew to manually perform such surveys through EVA alone.

Since 2006, therefore, we have been developing a system for performing systematic site surveys with multiple robots. ${ }^{13,14}$ With our approach, robots can be coordinated from ground-control or from inside surface habitats (or vehicles). A typical scenario involves multiple robots autonomously mapping a region while human operators assess the collected data and remotely intervene when necessary.

\section{A. Robotic Survey System}

Our robotic survey system involves three phases: planning, execution, and analysis. In the planning phase, we designate survey points and intermediate waypoints (to ensure that specific areas are visited or avoided) using satellite images. Depending on survey instrument, we may also compute survey paths using a semiautomatic coverage planner, which considers instrument constraints (e.g., field of view), spatial coverage requirements (e.g., parallel-line transects), and robot navigation concerns (max slope, drive speed, etc).

In the execution phase, we use a on-board task executive to control robot actions (navigation to waypoints, survey data collection, etc.), monitor execution, and respond to contingencies. Throughout this process, no off-board communication is required. This enables survey operations to be robust in the presence of intermittent data network failures, operator inattention, etc. 
In the analysis phase, we generate instrument-specific data products (e.g., $3 \mathrm{D}$ terrain models from range data) and summarization of robotic survey performance. To date, much of our field work has involved topographic mapping using 3D scanning lidar. Thus, we routinely generate 3D terrain models by processing lidar point-cloud data. To summarize robot performance, we have begun developing a system (Figure 4) that automatically computes a variety of metrics (time on task, mean time between interventions, etc.) and alerts users to key events when they occur. ${ }^{15}$

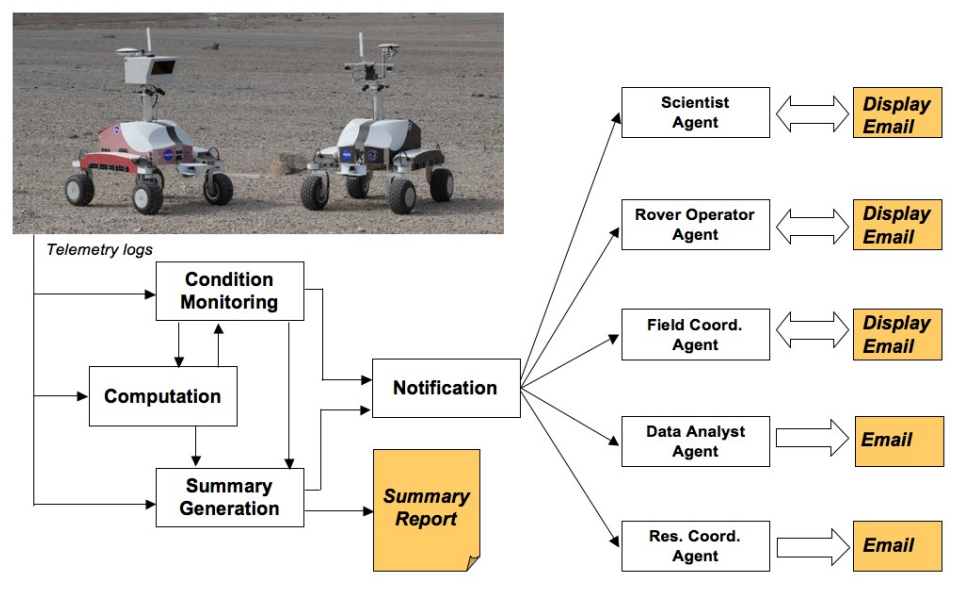

Figure 4. Summarization and event notification architecture

\section{B. Remote Operations}

We employ a variety of graphical user interfaces for site survey monitoring and robot control. We use Google Earth to display survey plans and monitor survey progress (with real-time updates) in wide-area geospatial context. We use Viz to show robot state (position, health, etc.) and real-time "snapshots" of survey data (useful for verifying that survey instruments are functioning properly).

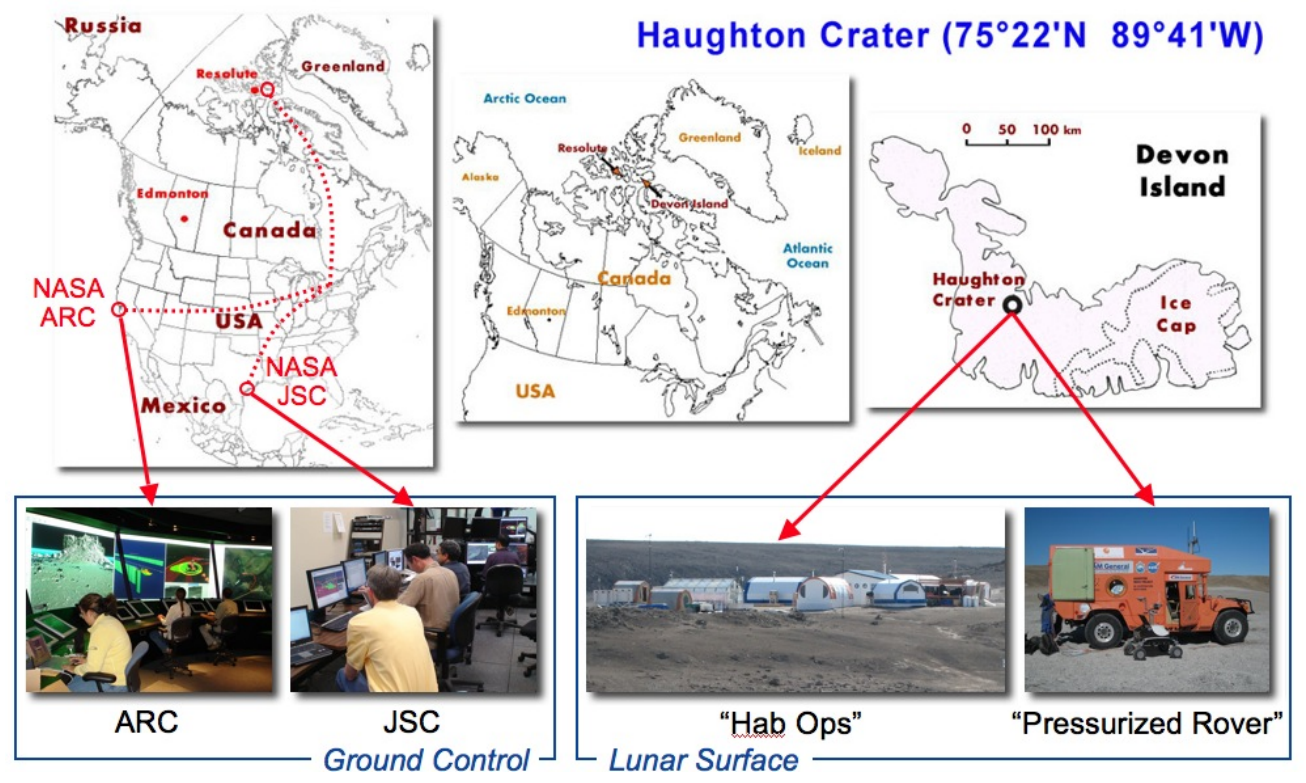

Figure 5. Robotic survey remote operations: left, "ground control" mode; right, "lunar surface" mode

With our system, survey robots can be remotely operated in two modes (Figure 5): (1) "ground-control" (with variable communications delay and limited bandwidth) or (2) from inside habitats or vehicles (with minimal delay and high bandwidth) as might be done on the lunar surface.

\section{Field Tests}

\section{2007 Haughton Crater Field Test}

In July 2007, we used two K10 rovers to map several sites at Haughton Crater, Devon Island, Canada ${ }^{14}$ as part of the annual Haughton-Mars Project (HMP) field season. Haughton Crater is a $20 \mathrm{~km}$ diameter impact structure and is similar in scale to Shackleton Crater, one of the primary candidate sites for a lunar outpost. K10 Black was equipped with the JPL CRUX ground-penetrating radar (GPR) ${ }^{16}$ for subsurface mapping. K10 Red was equipped with an Optech ILRIS-3D scanning lidar for topographic mapping. 

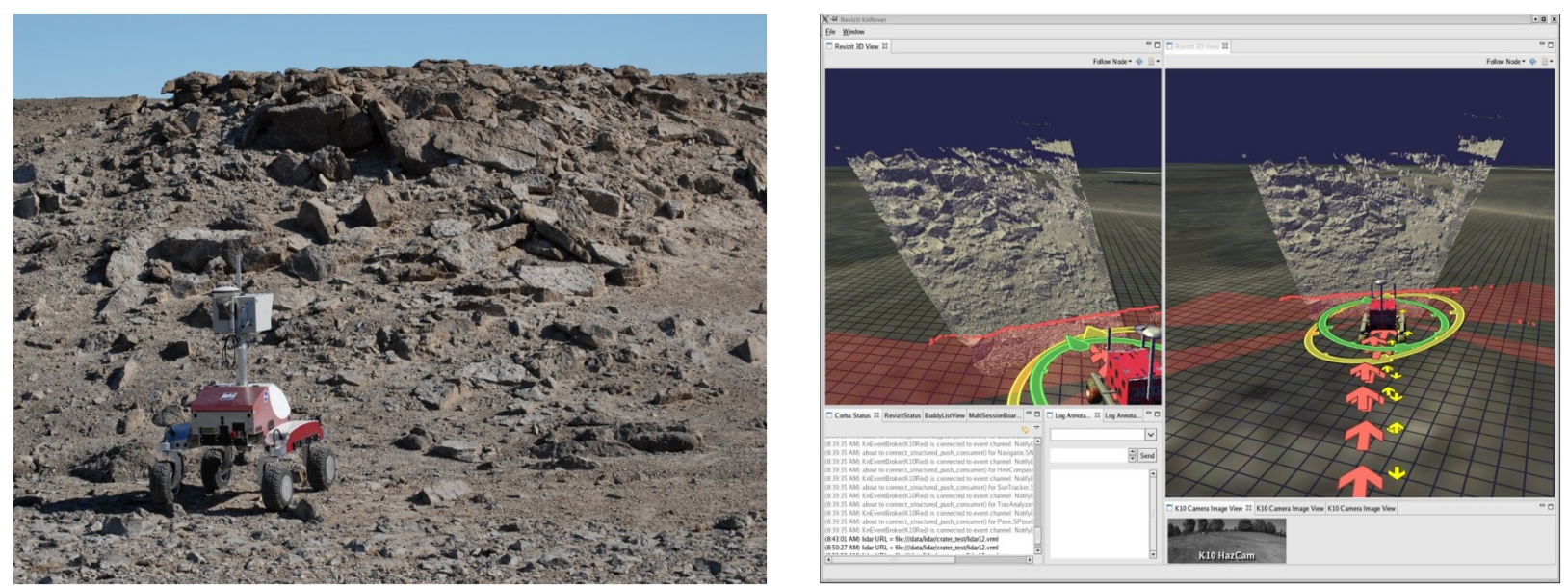

Figure 6. Left, K10 Red surveying a rocky slope; right, Viz showing real-time telemetry and a 3D lidar scan.

During the three-week field test, we performed more than 200 hours of robotic survey operations (Figure 6). Ten percent of these operations were conducted while the K10's operated outside of communication range (i.e., fully autonomously). K10 Red operated for 9 days, driving $14 \mathrm{~km}$ and collecting 25 lidar panoramas. K10 Black operated for 10 days, driving $32.2 \mathrm{~km}$.

As part of the test, rover operations simulated a near-term lunar mission, including the use of orbital data, interactive robot user interfaces, and remote operations procedures for intra-vehicular activity (IVA) and ground-control. We conducted surveys at several sites, which were selected to represent a variety of lunar terrains in terms of slope, composition, scale and remoteness. One survey focused on mapping "Drill Hill": a 700x700 m region located some distance from the HMP base camp.
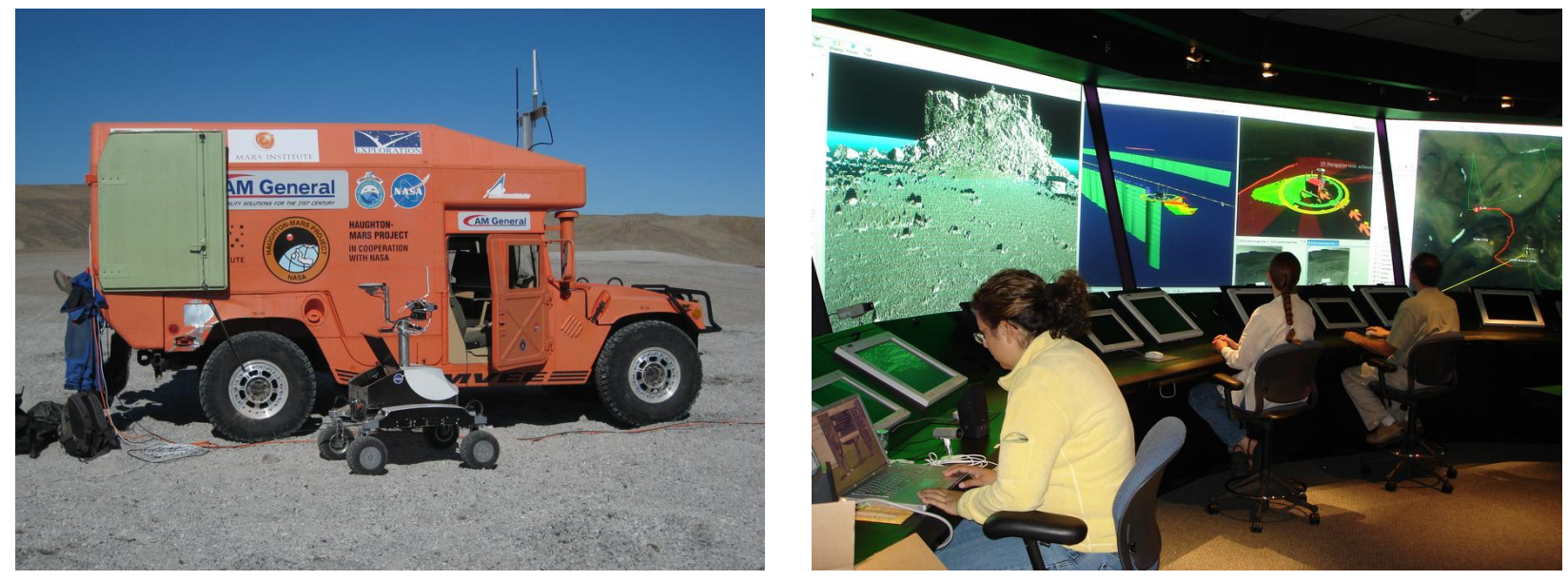

Figure 7. Left, A HMMWV was used to simulate a "small pressurized rover" for the Drill Hill survey; right, Simulated ground-control team at NASA Ames.

To simulate lunar surface sortie operations in a small pressurized rover, we stationed a HMMWV on Drill Hill (Figure 7, left) and used laptops as control stations, operating on-site for four days. To test ground control operations, we set up a remote data link (including a short satellite hop) between the HMP base camp and NASA Ames. A three-person team at NASA Ames (Figure 7, right) monitored K10 Black and K10 Red survey performance via the remote link and processed lidar data to build 3D terrain models.

\section{2008 Moses Lake Sand Dunes}

In June 2008, we used K10 Black to examine subsurface structure at Moses Lake Sand Dunes, Washington as part of the 2008 NASA Human-Robotic Systems Project field test. To do this, we equipped K10 Black with a GSSI SIR-3000 GPR and a $900 \mathrm{MHz}$ antenna. During the test, we drove K10 Black on both cross-country traverses and on parallel-line transects (see Figure 8). 


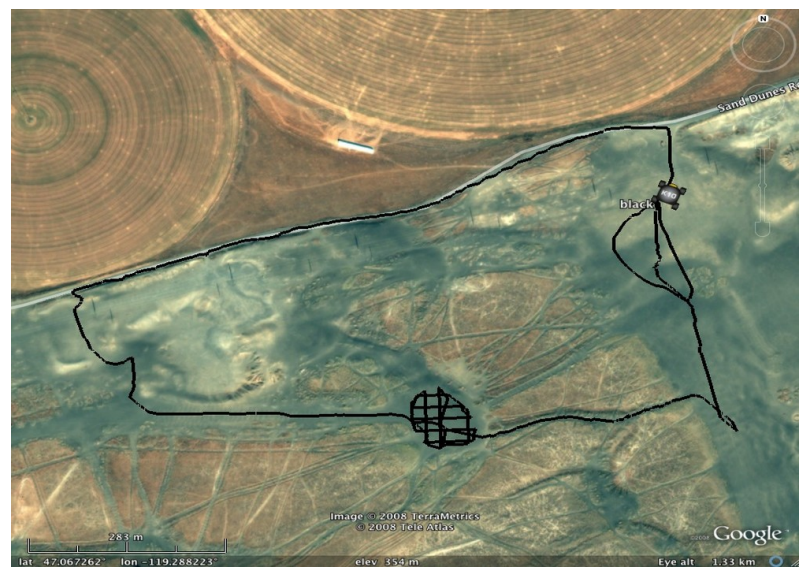

Figure 8. Paths driven by K10 Black while collecting GPR data at Moses Lake Sand Dunes

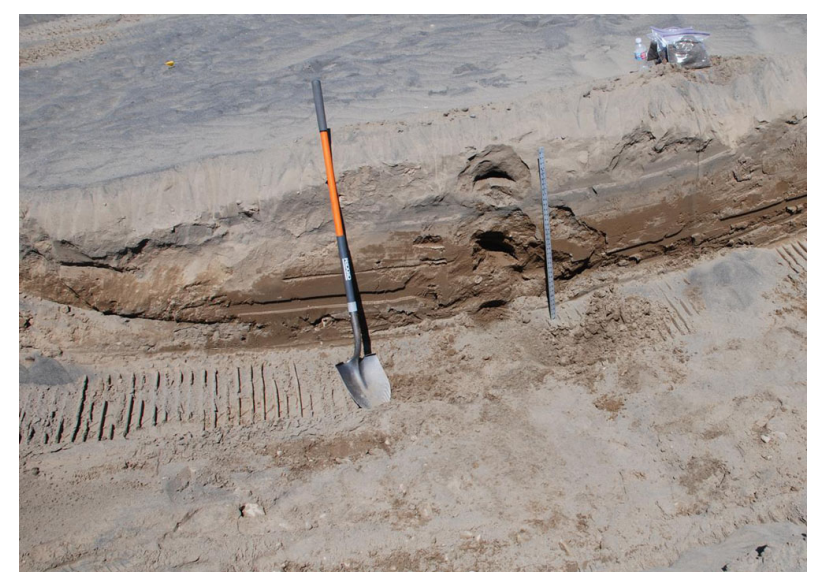

Figure 9. Excavated trench reveals layers of wet clays and alluvium beneath Moses Lake Sand Dunes

The GPR data collected by K10 revealed several features of the subsurface and the formation mechanisms of the sand dunes. The $900 \mathrm{MHz}$ scans showed subsurface layering with different backscattering coefficients to depths of 1 to $2 \mathrm{~m}$. The variation in depth penetration was due to different levels of clay and moisture in the ground material. By analyzing the number and angles of the subsurface layers, we believe that it is possible to estimate dune size at earlier dates.

At one point during the test, real-time analysis of the GPR data revealed layering that appeared to be a moisture gradient. To verify this, we excavated a $1 \mathrm{~m}$ deep trench (Figure 9) and examined the subsurface. Below the top layer of dry sand $(20 \mathrm{~cm})$, we identified layers of wet clays and alluvium. This suggests that the sand dune complex contains materials derived from water sedimentation. If we had only considered visible surface features and samples of the areas, we might have have concluded that Moses Lake is a desertic zone, which the subsurface layers show it clearly is not.

\section{Resource Prospecting}

Although orbital remote sensing can provide much information about the Moon, resource prospecting requires mapping work to be performed on the lunar surface. In particular, assessment of subsurface resources will require sample extraction (excavation, drilling, etc.), sample handling, and proximal (or contact) measurements.

For example, the Lunar Prospector orbiter detected significant quantities of hydrogen at the Lunar poles, but the form and concentration of the hydrogen is unknown (solar wind hydrogen, water ice, or hydrates). Identifying and quantifying the distribution (both horizontal and vertical) of these deposits is essential for determining if such resources can support long-duration lunar exploration. To do this, assessment will need to be performed to depths of (at least) $0.5 \mathrm{~m}$ in permanently shadowed regions.

To study how robots can be used for lunar resource prospecting, we have integrated the HYDRA neutron spectrometer (Los Alamos National Laboratory) and a Californium-252 source on to a K10 rover. ${ }^{17}$ In late 2007, we began conducting a series of controlled field trials to detect and map targets of various hydrogen contents and burial depths.

\section{A. Neutron Detection}

On the lunar surface, galactic cosmic rays constantly impinge on the regolith and produce high-energy neutrons $(\sim 10 \mathrm{MeV})$ through nuclear reactions. The high-energy neutrons lose energy either by scattering within the soil, or are absorbed by neutron capture reactions. At equilibrium, the neutron flux can be considered to be divided into thermal neutrons (0.01 to $0.4 \mathrm{eV}$ ), epithermal neutrons $(0.4 \mathrm{eV}$ to $0.5 \mathrm{MeV}$ ) and fast neutrons (0.5 to $10 \mathrm{MeV}$ ).

When hydrogen is present in soil, elastic scattering provides an efficient mechanism that reduces the energy of epithermal neutrons. In particular, enhanced hydrogen deposits facilitates moderation and thermalization of energetic neutrons, and a decrease (of up to two orders of magnitude) in the leakage flux of epithermal neutrons induced by cosmic-rays. Thus, by measuring the count rates of thermal and epithermal 
neutrons, we can estimate the depth and abundance of hydrogen contained in the soil.

The key challenge to doing this with a rover-mounted instrument is achieving acceptable statistics given instrument geometry (e.g., distance above ground) and rover motion (speed and measurement dwell time). For example, to achieve $\pm 10 \%$ uncertainty (10 wt \% water-equivalent hydrogen) requires at least 100 counts to be obtained for any given measurement station.

\section{B. HYDRA Integration}

On the lunar surface, cosmic rays constantly impinge on the regolith, but for terrestrial testing, a Californium-252 neutron source (activity of $\sim 2 \times 10^{6}$ neutrons/s) is used to interrogate the soil beneath the rover. The source was co-located with the HYDRA instrument, to provide a fixed geometry and to minimize the effects of variations in soil-to-source distance.

Figure 10 shows HYDRA and the source mounted on the front of K10 Black. When the energetic neutrons from the source encounter hydrogenous materials, HYDRA measures an enhanced backscatter "albedo" flux of thermal and epithermal neutrons.

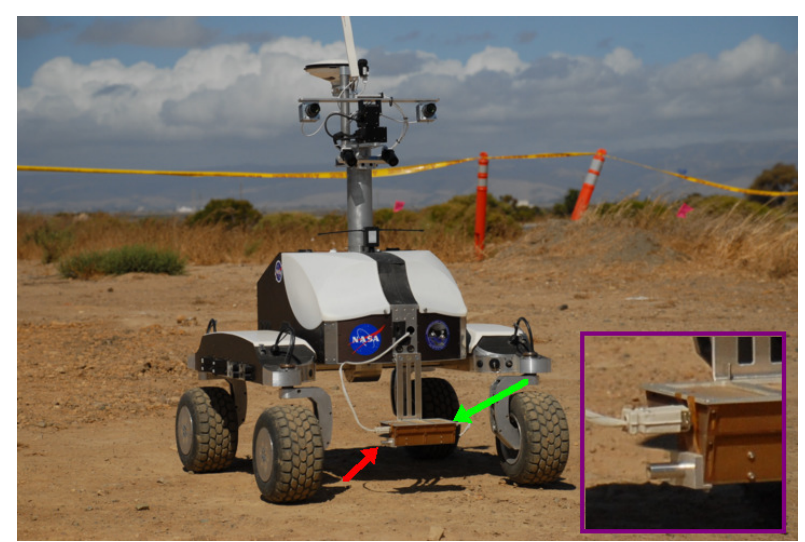

Figure 10. K10 Black with HYDRA mounted (green arrow) and neutron source (red arrow). Inset, close-up of the source and HYDRA cable harness.

\section{2007 HYDRA test at NASA Ames}

In September 2007, we conducted an initial test at NASA Ames with K10 Black and HYDRA to demonstrate the utility of robotic prospecting for near-surface hydrogenous deposits. A relatively level, unvegetated pad of fill dirt measuring $50 \mathrm{~m} \times 100 \mathrm{~m}$ was used for the test. Within this area, buried sheets of polyethylene and gypsum served as proxies for hydrous minerals and interstitial (pore) ice. The locations of the polyethylene and gypsum targets were known only to three personnel involved in the exercise, in order to provide a single-blind test.

Overall, we found that a coarse-fine survey strategy is an appropriate method for hydrogen prospecting. The primary factors that influence the success of using robots for this function are: (1) selection of rover traverse speed that balances time of operations with spectrometer spatial resolution (high rover speeds results in low resolution), (2) sensor field of view (e.g., the current K10/HYDRA configuration limits detection range to $50 \mathrm{~cm}$ radially), and (3) sufficient contrast between resource deposit and environment (i.e., surrounding regolith must have low hydrogen content).

\section{Calibration}

The first phase of the field test involved HYDRA calibration. To do this, we drove K10 across known locations of three targets at four speeds: 5, 10, 20, and $40 \mathrm{~cm} / \mathrm{s}$. Figure 11 (left) shows K10 Black during one of the calibration traverses. The right panel shows the HYDRA HeSn detector response when passing over a polyethylene calibration target.

Based on the data collected during these traverses, we determined that the rover speed should be $10 \mathrm{~cm} / \mathrm{s}$ for the transect survey. The $5 \mathrm{~cm} / \mathrm{s}$ speed provided excellent resolution, but required too much time to complete the survey. The $20 \mathrm{~cm} / \mathrm{s}$ and $40 \mathrm{~cm} / \mathrm{s}$ speeds did not satisfactorily resolve the smaller targets.

The calibration phase also revealed that the test site contained a surprisingly high hydrogen content: approximately $10 \mathrm{wt} \%$ water equivalent. This is roughly a factor of 100 times higher than what would be found in dry lunar regolith. As a result, we hypothesized that it would be difficult to locate all the buried targets, which was confirmed by subsequent testing.

\section{Coarse mapping}

After calibration, we performed coarse mapping by conducting a parallel-line transect survey with approximately $1 \mathrm{~m}$ separation between transects. Figure 12 (left) shows a map of K10's traverse. Because of the 

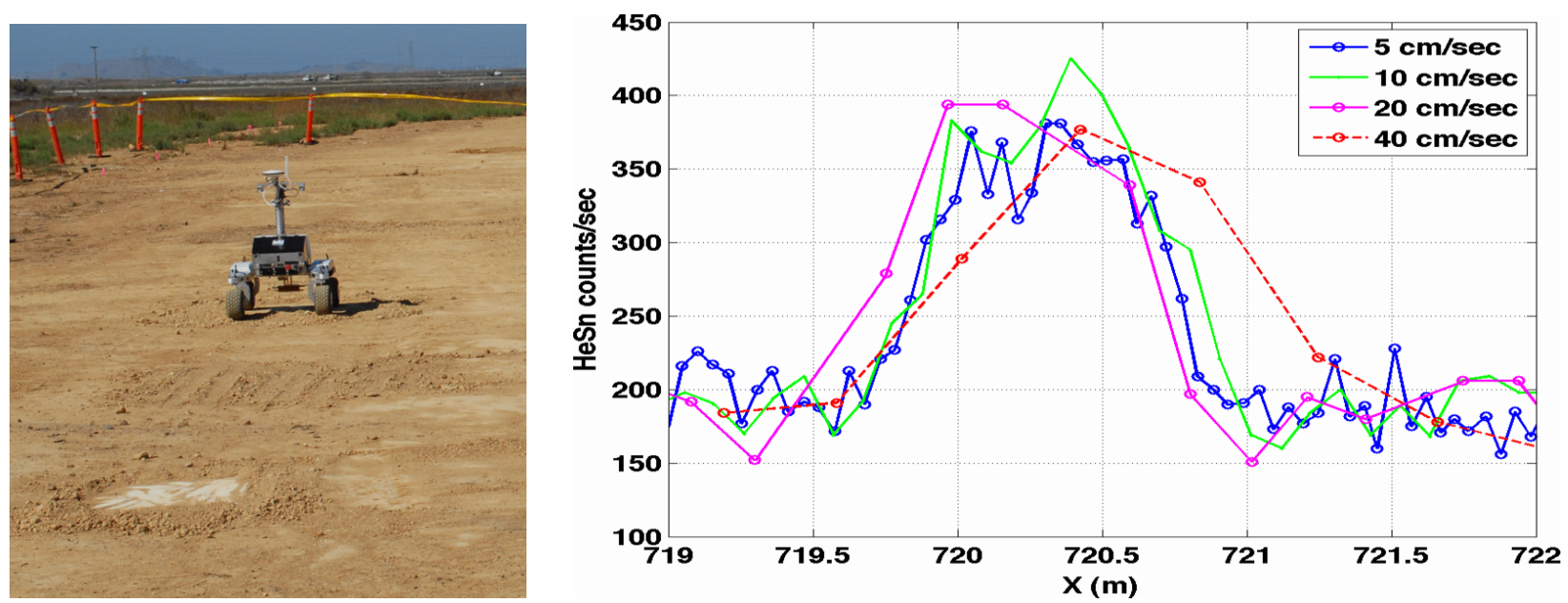

Figure 11. Left, K10 driving a calibration traverse. An exposed polyethylene sheet is visible in the foreground; right, comparison of sample spacing vs. traverse speed.

way the rover executed turns and headed toward subsequent waypoints, the transect spacing was sometimes greater than 1 meter.

Considerable information was obtained by HYDRA during the coarse survey. Figure 12 (right) shows maps of HeSn (thermal and epithermal) and HeCd (epithermal only) count rates, binned to 50x50 cm cells. Note that we have truncated the color scale at 250 counts/s for the HeSn map, in order to bring out the detail.
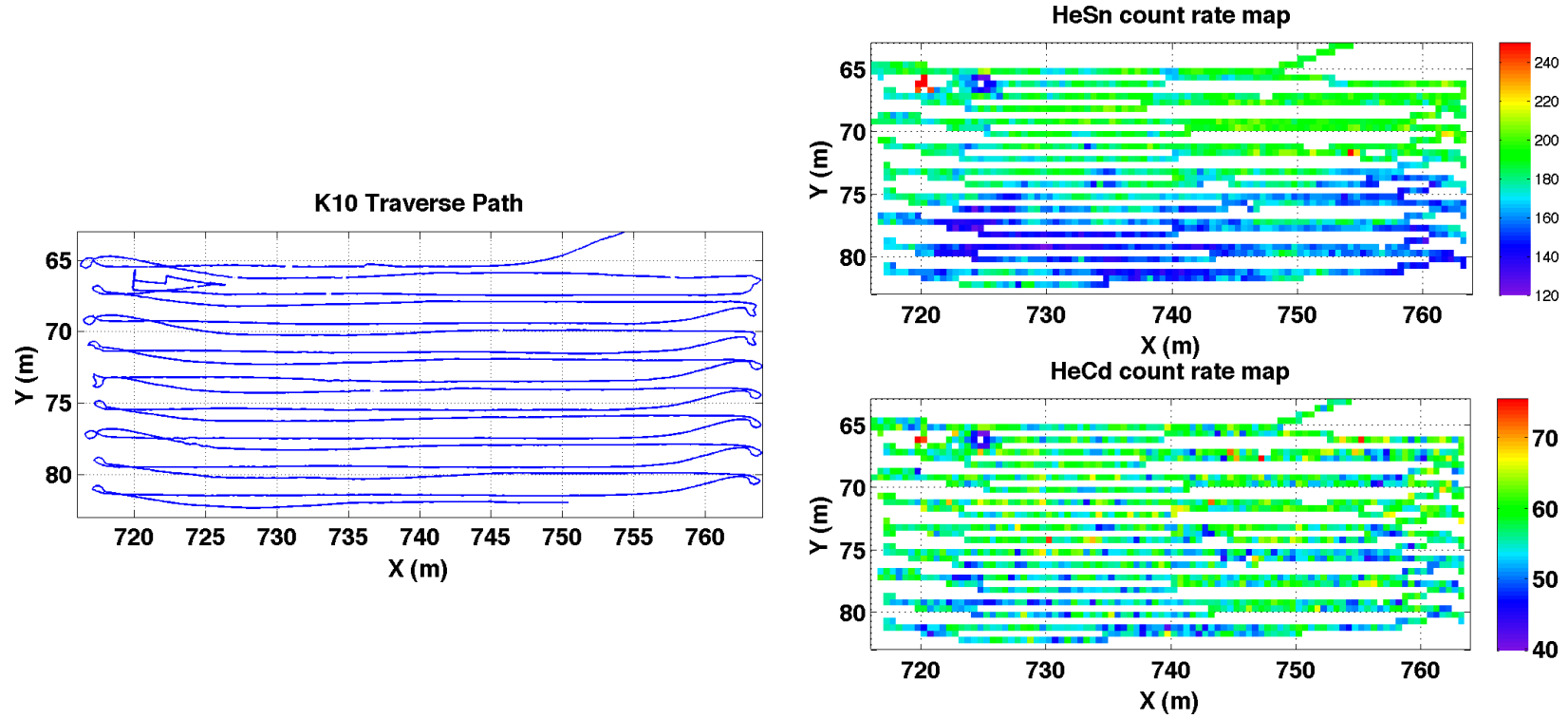

Figure 12. Left, K10 traverse path for the coarse mapping survey. The transect separations were planned to be $1 \mathrm{~m}$ apart; right, binned HeSn (thermal+epithermal) and HeCd (epithermal only) neutron count rates.

Two of the polyethylene calibration targets appear as highs on the HeSn map at $(720,66) \mathrm{m}$ and at $(754,72) \mathrm{m}$. The low on both the HeSn and HeCd map at $(725,66) \mathrm{m}$ corresponds to a deposit of dried Monterey beach sand, which has far less hydrogen than the surrounding soil. Finally, note that the lower region of the test area ( $\mathrm{Y}=75$ to $82 \mathrm{~m}$ ) appears to contain much less hydrogen than the upper part of the test area. A post-test geochemical analysis of material from the lower region revealed that it contains a higher concentration of $\mathrm{Fe}_{2} \mathrm{O}_{3}$, which absorbs thermal neutrons. 

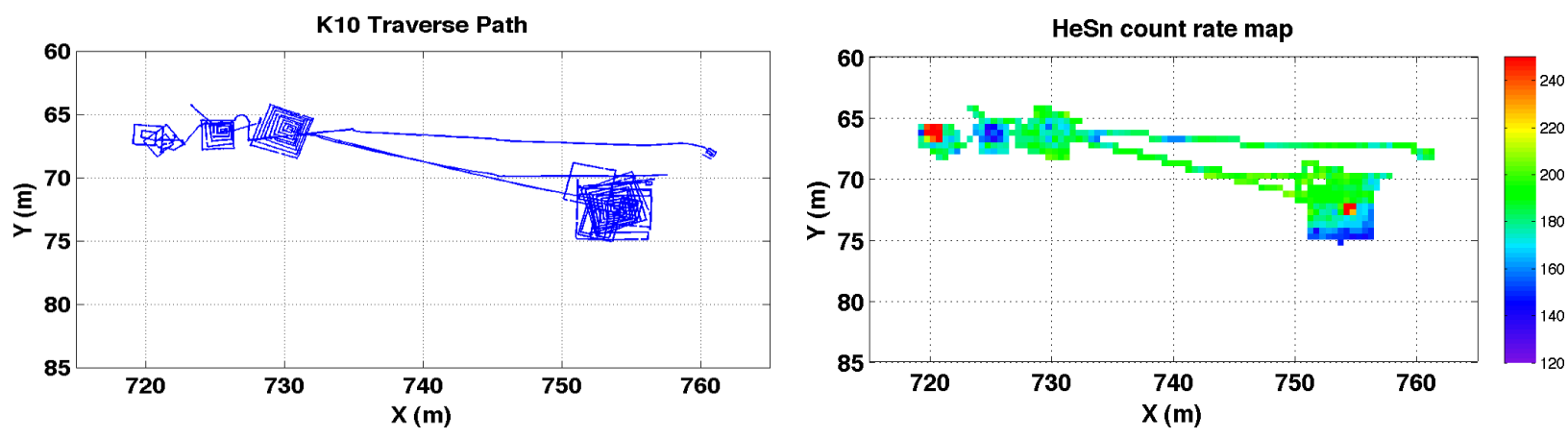

Figure 13. Top, K10 traverse path for fine characterization of specific features; bottom, binned HeSn (thermal+epithermal) neutron count rates.

\section{Feature Mapping}

In the third phase, we carried out a more detailed mapping of selected features identified by coarse mapping. We chose to include the calibration features in this study as a way of testing K10's ability to perform high spatial resolution investigations of small features. Also included were some of the interesting features detected elsewhere in the test area. In each case, K10 was directed to move to the center of a designated feature location and then carried out a rectangular spiral search pattern around the target. In this way, a higher sample density was obtained, permitting characterization of each feature's spatial extent.

Figure 13 shows that the target characterization traverses are dominated by spiral sampling patterns centered on the points of interest. The HeSn detector clearly detects and places limits on the hot spot of the polyethylene calibration target at $(721,66) \mathrm{m}$. To its right the dry sand target and the exposed gypsum targets show up as lower count rate areas. On the right, at $(754.5,72.5) \mathrm{m}$, a second hotspot is clearly revealed. This corresponds to a polyethylene target buried under $5 \mathrm{~cm}$ of soil.

\section{Robotic Recon}

NASA currently plans to return to the Moon with a campaign of surface missions spaced on six month intervals. ${ }^{18}$ A representative campaign ${ }^{18}$ is shown in Figure 14. Some early missions will consist of cargo-only landings to deliver surface assets including mobility systems, habitats, power, payload handling, etc. Others will be short duration (two week) human "sortie" missions with limited surface time. The overall objective is to build up surface infrastructure and operational capability over time, leading to the establishment of a full-scale lunar outpost.

Prior to these surface missions, spacecraft in lunar orbit will be used to map the surface. These orbital missions will provide visible image base maps, topographic maps, gravity maps, etc. However, remote sensing data may not be of sufficient resolution, nor view angle, to fully plan lunar surface activity, such as crew traverses for field geology. Thus, it will be important to acquire supplemental and complementary data on the lunar surface.

One way to do this is through robotic reconnaissance, i.e., using a planetary rover to scout traverses, or sites, prior to EVA activity. Such recon work can be done before, or between, human missions. A key benefit is that data acquired by a planetary rover can provide significant improvements in resolution $m m$ to $\mathrm{cm}$ scale, rather than $m$ to $\mathrm{km}$ scale, along with oblique viewpoints not achievable from space. In addition, recon can retire risk by helping identify operational considerations (e.g., surface hazards).

Our approach to robotic recon makes use of three types of sensors, which we have integrated on to K10 Red. The first is a 3D lidar (Optech ILRIS-3D) which provides $\mathrm{cm}$ to $m$ resolution measurements of terrain. This represents more than two orders of magnitude higher resolution than will be available from the Lunar Orbital Laser Altimeter (LOLA). Second is a consumer-grade digital camera mounted on a pan/tilt unit, which is used to capture high-resolution, color panoramas ( $60^{\circ}$ vertical and $135^{\circ}$ horizontal field-of-view). In the near field, these panoramas provide several orders of magnitude higher resolution than the Lunar Reconnaissance Orbiter Camera (LROC), as well as surface views. Third is a microscopic imager with $70 \mu \mathrm{m}$ per pixel images, which providing very high-resolution images of surface materials. 


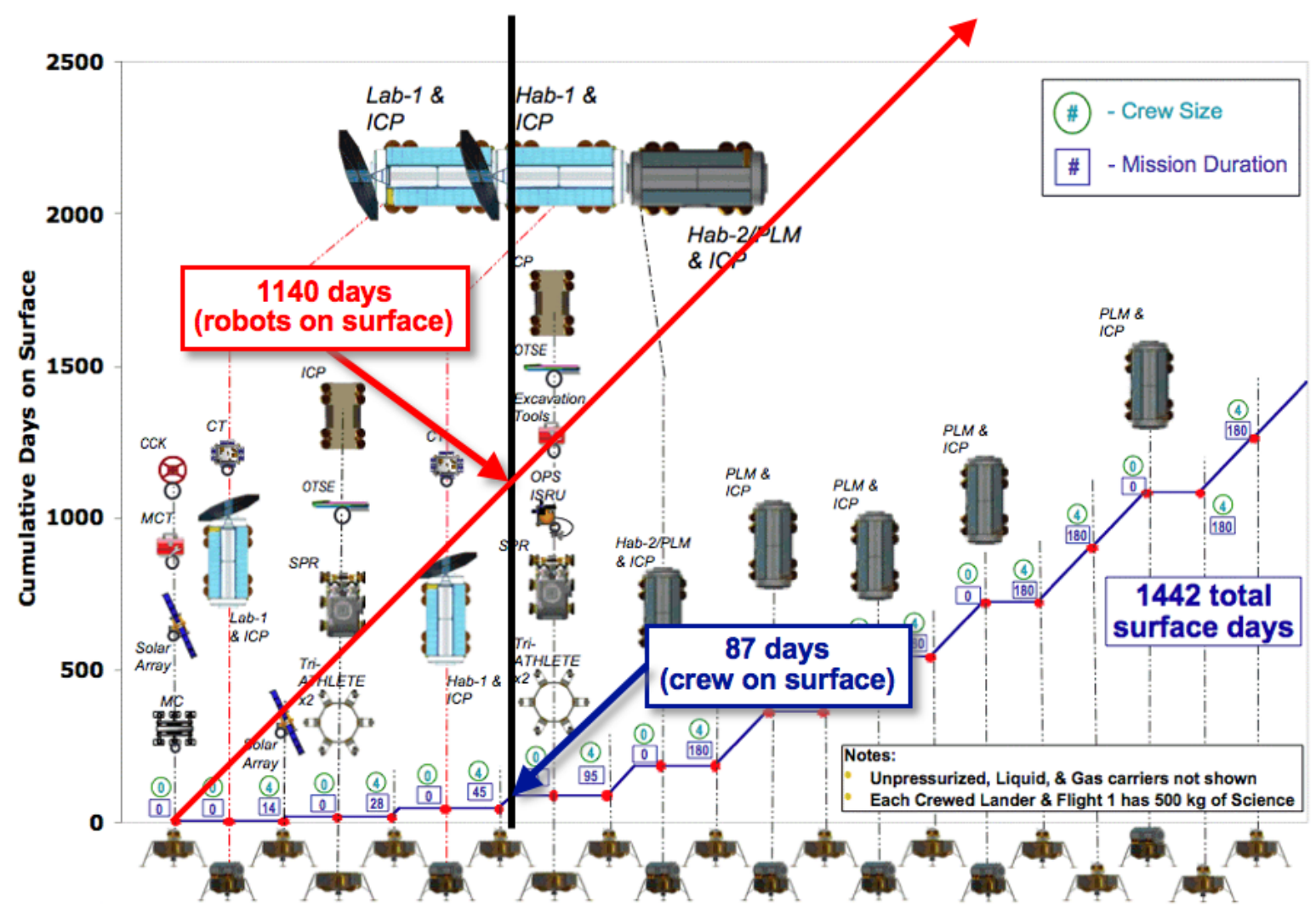

Figure 14. Notional lunar campaign. After the first three years, human crews have accumulated less than 90 days on the surface, while surface assets including a mobility chassis have more than 1,100.

\section{A. Experimental Ground Control}

Our operations concept is derived from lessons learned by Apollo, Space Shuttle, Space Station, and the Mars Exploration Rovers. Each of these NASA mission/program examples have overlap with the objectives and operational constraints we anticipate for lunar surface robots. ${ }^{19}$ Operations requirements are affected by differences caused by surface vs. orbit locations, known vs. unknown and structured vs. unstructured environments, low vs. high latency data, low vs. high bandwidth, frequent vs. infrequent communications, manned vs. unmanned spacecraft, etc.

Operational roles are split according to the timelines on which they operate (Figure 15). The prioritization of scientific objectives or analysis of data occur on what we refer to as Strategic timelines. Strategic timelines also include the establishment of

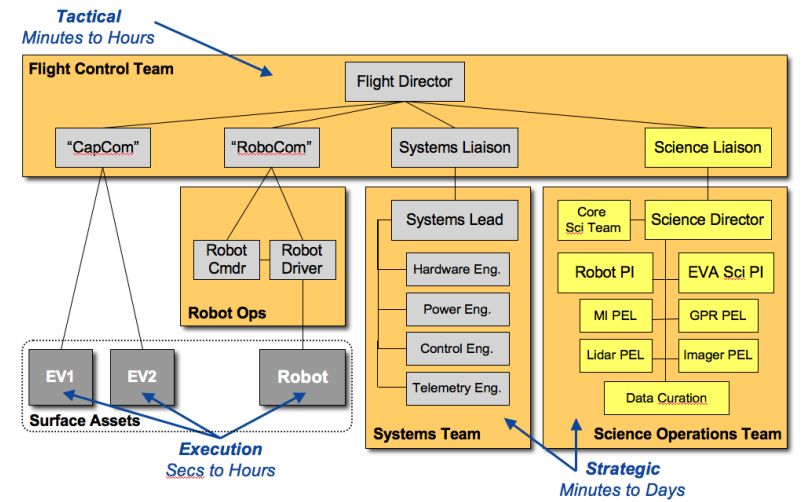

Figure 15. Our experimental ground control structure. The Science Team functions similarly to the MER SOWG. The Flight Control Team functions similarly to the current Space Station mission control. long term flight rules, engineering objectives, and monitoring of robot health and performance. On the Tactical timeline, the flight controllers interact minute-by-minute with the Flight Director, address problems and resolve conflicts with representatives from the major systems and stakeholders in the mission, such as the science team. On the Execution timeline, the robot controllers interact in real-time with the robot over a few seconds of data transmission delay, and the robot closes the loop on executing uploaded command sequences. 
Our ground control includes the real-time, tactical operations structure of Apollo and Space Station (a core Flight Control Team led by a Flight Director) combined with the strategic timelining, analysis, and decision making of the Mars Exploration Rover (MER) Science Operations Working Group (SOWG), i.e., a Science Team led by a Science PI. The Science Team interacts with the Flight Control Team through a Science Officer Liaison. ${ }^{20}$ During EVA activity, the Science Team provides support as a "science backroom" and interacts with crew via a "CapCom" officer. In the future, the Science Team may also interact directly with crew as recommended by. ${ }^{21}$

To facilitate the science team functions, we developed a robot traverse planning tool called GeOps, shown in Figure 16. GeOps provides a GUI for interfacing with the Google Earth client to establish a series of robot traverse waypoints with associated data collection tasks. The user interface provides a list view and timeline view of the plan, and a $\mathrm{KML}^{22}$ network link provides a map view of the plan in Google Earth. Because the traverse planning tool uses a platform already familiar to the science team, they were able to use it effectively with very little training.

Data collected during robotic recon is automatically processed by our ground data system. Each data product includes spatial location indicating

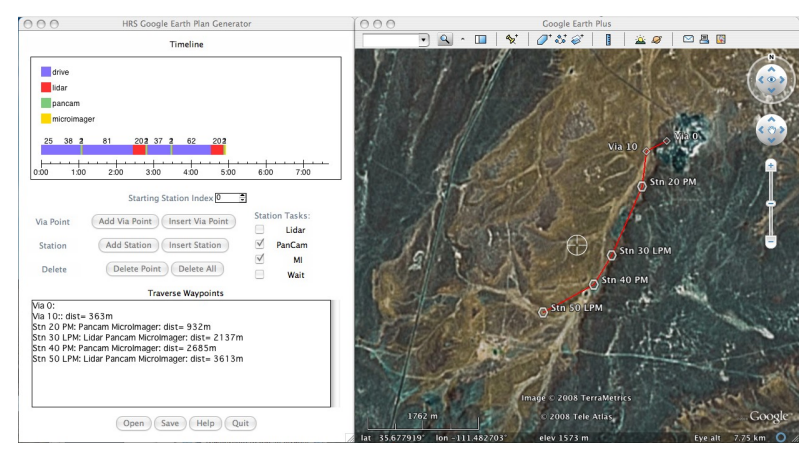

Figure 16. GeOps robot traverse planning tool provides an interface to Google Earth, a familiar tool to scientists, as well as a list view and timeline view of the plan as it is manually constructed.

where the data was collected. This is converted to a KML placemark for spatial browsing of the data, with a link to the full data product in the archive. The archive is implemented using the open-source Gallery photo management system, which leverages familiar photo album sorting and browsing functions to organize our rover data for ease of use. 3D data is visualized using Viz. ${ }^{10}$

\section{B. 2008 Moses Lake Sand Dunes Field Test}

In June 2008, we conducted an initial test of robotic recon approach as part of the 2008 NASA HumanRobotic Systems Project field test at Moses Lake Sand Dunes. By 2025, we expect that robots will operate on the lunar surface for six months between human missions, which will last for two weeks. Thus, to approximate the ratio between periods of robot and human activity, we operated the K10 Red rover in robotic recon mode for two days at Moses Lake followed by a one hour crew EVA.

The robotic recon experiment included several phases:

1. Remote sensing data were used to identify targets of interest.

2. The Science Team planned robotic traverses to visit areas of interest for closer inspection.

3. The plans were vetted by the Flight Control Team, and when all systems were ready, the Robot Control Team executed the traverse plan. We operated K10 in both autonomous and teleoperated modes.

4. Data returned from the robot were automatically organized into the Ground Data System. Geocoded tags on a map showed data products taken at specific locations. Hyperlinks provided a connection from those placemarks to the data products in a web archive.

5. The Science Team analyzed data in the archive to improve its understanding of the test site geology, as well as to create a prioritized list of stations for subsequent EVA activity.

6. An EVA planning team used the list of stations with science objectives, operational constraints (i.e, "flight rules") and timeline constraints to produce an EVA plan.

7. EVA crew were briefed on the EVA plan and provided "cuff checklists".

8. The EVA crew carried out the EVA with real-time support from the Science Team via a "CapCom" on a voice loop. 
The usefulness of robotic recon was subjectively evaluated by the science team throughout the test. Specifically, the team considered how their understanding of the site and of the science objectives to be achieved changed as additional recon data were acquired. The team observed that data from the robot filled in significant gaps in their understanding (based originally only on remote sensing) of site features and scale, vegetation, mineralogy, grain size distribution, and variations in surface albedo.

For sites that were visited by the robot, the science team obtained detailed knowledge through direct observation. But, even for sites that the robot did not visit, the team was able to extrapolate their knowledge to generate a reasonable expectation for what would be found. Furthermore, the science team concluded that the robotic recon data was extremely helpful for EVA traverse planning. Finally, the science team recommended that follow-up field work should be performed at the test site to determine what might have been missed (or misinterpreted) by robotic recon.

Overall, our initial test revealed that robotic recon serves a very different function than robotic exploration, such as performed by MER. Where as robot explorers serve as the primary tool for science (i.e., exhaustively studying a sample, station, or site before moving on), the purpose of recon is to high-grade a site in order to triage and prioritize stations, or targets, for subsequent human activity. Thus, if a feature of significant interest is discovered during recon, the location is marked and the robot is sent on to scout additional areas.

\section{Future Research}

Looking forward, we will continue to develop utility robots to support several activities that can be performed even when humans are not on the lunar surface. Two tasks that we plan to address in the near-term are geotechnical assessment and shallow regolith excavation. Both tasks are ideally suited for lunar utility robots because they can be performed in a continuous manner, do not require human-paced interaction, and are amenable to automation.

There are numerous methods for assessing physical properties of soil. One widely used approach is to use a cone penetrometer to measure properties, such as density, as a function of depth. These measurements provide data for designing structural foundations, predicting trafficability, and testing soil/vehicle interactions. In the lunar environment, however, manually pushing a penetrometer into regolith is not practical because the soil resistance quickly exceeds the mass reaction of an astronaut.

In Spring 2009, we will integrate a Percussive Dynamic Cone Penetrometer (PDCP) on K10. The PDCP overcomes the difficulty of deploying a conventional penetrometer in low gravity by using a percussive device, such as a hammer drill, to successively impact the drive shaft. By measuring shaft penetration vs. number of impacts, it is possible to assess bearing strength, soil compaction, and other mechanical properties. ${ }^{23}$

Numerous methods have been proposed for excavating lunar regolith. For surface work, one novel method is to use a monopropellant (or other gas) to pneumatically excavate using positive pressure. This would enable both traverse mining and plunge mining to be performed at lower reaction forces than with conventional (i.e., blade/scoop) systems. It is estimated that $1 \mathrm{~kg}$ of monopropellant could excavate 6 to 9 tons of regolith.

During the next year, therefore, we plan to investigate how to integrate and operate a pneumatic excavator on a planetary rover. We anticipate that several key issues will have to be addressed, including rover traction, manipulation and control of the gas dispensing device, and dust management. Understanding these issues will almost certainly involve low-pressure (less than 1 torr) testing with lunar regolith simulant.

In addition to these two tasks, there are numerous other utility robot functions that we believe are worth developing. These include: mobile camera platform (to provide supplemental camera views for payload handling, maintenance, etc.), communication network mapping (to identify "dead zones" and maximum reliable range"), communications relay deployment (to provide "fill-in" or temporary coverage), traverse assessment (to verify trafficability), and navigational aid (to provide a reference for surface operations).

\section{Acknowledgments}

We thank Javier Barreiro, Vijay Baskaran, Vandi Verma and Steve Wragg for assisting with PLEXIL and the Universal Executive. We sincerely thank the Desert Research and Technology Studies team for their support at Meteor Crater and the Mars Institute for its support at Haughton Crater. We gratefully acknowledge the assistance of David Kring and Essam Heggy with K10 studies at Moses Lake Sand Dunes. This work was supported by the NASA Exploration Systems Technology Development Program under the "Human-Robotic Systems" and "In-Situ Resource Utilization" projects and by the NASA Innovative Partnership Program. 


\section{References}

${ }^{1}$ Bualat, M., Kobayashi, L., Lee, S., and Park, E., "Flexible Rover Architecture for Science Instrument Integration and Testing," Space, AIAA, 2006.

${ }^{2}$ Flückiger, L., To, V., and Utz, H., "Service Oriented Robotic Architecture Supporting a Lunar Analog Test," International Symposium on Artificial Intelligence, Robotics, and Automation in Space, 2008.

${ }^{3}$ OMG, "Object Management Group Interface Definition Language," [Online] http://www.omg.org/gettingstarted/omg idl.htm, 2006.

${ }^{4}$ OMG, "CORBA Frequently Asked Questions," [Online] http://www.omg.org/gettingstarted/corbafaq.htm, 2006.

${ }^{5}$ Nesnas, I., Wright, A., et al., "CLARAty and Challenges of Developing Interoperable Robotic Software," International Conference on Intelligent Robots and Systems, IEEE, 2003.

${ }^{6}$ Bualat, M., Edwards, L., et al., "Autonomous Robotic Inspection for Lunar Surface Operations," Field and Service Robots, Springer, 2007.

${ }^{7}$ Verma, V., Jonsson, A., et al., "Universal Executive and PLEXIL: Engine and Language for Robust Spacecraft Control and Operations," Space, AIAA, September 2006.

${ }^{8}$ Dowek, G., Munoz, C., and Pasareanu, C., "A Formal Analysis Framework for PLEXIL," Third Workshop on Planning and Plan Execution for Real-World Systems, 2007.

${ }^{9}$ Reinhard, E., Ward, G., et al., High Dynamic Range Imaging: Acquisition, Display, and Image-Based Lighting, Morgan Kaufmann, San Francisco, 2006.

${ }^{10}$ Edwards, L., Bowman, J., et al., "Photo-realistic Terrain Modeling and Visualization for Mars Exploration Rover Science Operations," International Conference on Systems, Man, and Cybernetics, IEEE, October 2005.

${ }^{11}$ Norris, J., Powell, M., et al., "Mars Exploration Rover Operations with the Science Activity Planner," International Conference on Robotics and Automation, IEEE, April 2005.

${ }^{12}$ Diftler, M., Ambrose, R., et al., "Crew/Robot Coordinated Planetary EVA Operations at a Lunar Base Analog Site," 38th Lunar and Planetary Science Conference, Lunar and Planetary Institute, 2007.

${ }^{13}$ Fong, T., Bualat, M., et al., "Human-Robot Site Survey and Sampling for Space Exploration," Space, AIAA, 2008.

${ }^{14}$ Fong, T., Allan, M., et al., "Robotic Site Survey at Haughton Crater," 9th International Symposium on Artificial Intelligence, Robotics, and Automation in Space, 2008.

${ }^{15}$ Schreckenghost, D., Fong, T., et al., "Human Supervision of Robotic Site Surveys," Conference on Human/Robotic Technology and the Vision for Space Exploration (STAIF), 2008.

${ }^{16}$ Kim, S., Carnes, S., et al., "Miniature Ground Penetrating Radar, CRUX GPR," Aerospace Conference, IEEE, 2008.

${ }^{17}$ Elphic, R., Utz, H., et al., "Preliminary Results of Hydrogen Prospecting with a Planetary Rover," 39th Lunar and Planetary Science Conference, Lunar and Planetary Institute, 2008.

${ }^{18}$ Culbert, C., "Lunar Surface Systems," 3rd Space Exploration Conference, AIAA, February 2008.

${ }^{19}$ Fong, T., Deans, M., et al., "A Preliminary Examination Of Science Backroom Roles And Activities For Robotic Lunar Surface Science," Lunar Science Conference, No. 2142, NASA Lunar Science Institute, 2008.

${ }^{20}$ Osborn, J., The Role of the Science Officer Flight Controller in the Upcoming Era of Lunar Exploration, NASA JSC Whitepaper, 2006.

${ }^{21}$ Engle, M., "Operational Considerations for Manned Lunar Landing Missions Lessons Learned From Apollo," Space, No. AIAA-2004-6081-506, AIAA, 2004.

${ }^{22}$ OGC, "OpenGIS KML Encoding Standard," Tech. Rep. OGC 07-147r2, Open Geospatial Consortium, 2008.

${ }^{23}$ Zacny, K., Fong, T., et al., "Percussive Dynamic Cone Penetrometer for Geotechnical Surface with a Planetary Rover," Lunar Science Conference, No. 2138, NASA Lunar Science Institute, 2008. 